\title{
Designing lattice structures with maximal nearest-neighbor entanglement
}

\author{
J. C. Navarro-Muñoz ${ }^{1}$, R. López-Sandoval ${ }^{1}$, and M. E. García ${ }^{2}$ \\ ${ }^{1}$ Instituto Potosino de Investigación Científica y Tecnológica, \\ Camino a la presa San José 2055, \\ 78216 San Luis Potosí, Mexico and \\ 2 Theoretische Physik, FB 18, Universität Kassel and Center for \\ Interdisciplinary Nanostructure Science and Technology (CINSaT), \\ Heinrich-Plett-Str.40, 34132 Kassel, Germany
}

\begin{abstract}
In this work, we study the numerical optimization of nearest-neighbor concurrence of bipartite one and two dimensional lattices, as well as non bipartite two dimensional lattices. These systems are described in the framework of a tight-binding Hamiltonian while the optimization of concurrence was performed using genetic algorithms. Our results show that the concurrence of the optimized lattice structures is considerably higher than that of non optimized systems. In the case of one dimensional chains the concurrence is maximized when the system begins to dimerize, i.e. it undergoes a structural phase transition (Peierls distortion). This result is consistent with the idea that entanglement is maximal or shows a singularity near quantum phase transitions and that quantum entanglement cannot be freely shared between many objects (monogamy property). Moreover, the optimization of concurrence in two-dimensional bipartite and non bipartite lattices is achieved when the structures break into smaller subsystems, which are arranged in geometrically distinguishable configurations. This behavior is again related to the monogamy property.
\end{abstract}

PACS numbers: 03.67.-a,03.65.Ud,73.43.Nq,71.10.Fd 


\section{INTRODUCTION}

Quantum entanglement is one of the most distinctive features in quantum mechanics and yet its properties are still not fully understood. This quantum resource is considered a key element for several quantum information and quantum computation proposals such as quantum teleportation ${ }^{\frac{1}{}}$, superdense $\operatorname{coding}^{2}$, certain kinds of quantum key distribution schemes and quantum secret sharing protocols ${ }^{3}, 4$.

Recently, much research has been focused on a better understanding of quantum correlations in multiparticle systems ${ }^{5}$,6,7,8. A characteristic property that sets apart quantum correlations (or entanglement) from the classical ones is that entanglement cannot be freely shared among many objects. For the special case of three qubits $A, B$ and $C$, this has been shown by Coffman et al $\stackrel{9}{\cdot}$ using concurrence, a measure of entanglement taking values between 0 and $1 \underline{10}$. Coffman et al. showed that the sum of the squared concurrences $A B$ and $B C$ cannot be greater than unity, meaning that the degree of entanglement between $A$ and $B$ limits the entanglement between $A$ and $C$. This property is called monogamy of entanglement. For an infinite chain of qubits sharing uniform entanglement with their first neighbors, Wootters ${ }^{6}$ found an upper limit for concurrence of $1 / \sqrt{2}$ (this limit, however, has not been yet proven for physical systems). Under certain conditions, Wootters reports a maximum concurrence of $\mathrm{C}_{\max }=0.434467$, a result which has also been confirmed in the case of rings with $N$ qubits when $N \rightarrow \infty^{\underline{\underline{5}}}$. Dür et al. $\underline{7}$ have shown that in the case of three qubits, average concurrence between pairs of qubits of the $|W\rangle$ state are all equal to 2/3, while Koashi et al. $\frac{8}{-}$ have reported a maximum average concurrence between all pairs of qubits of $2 / N$ when all qubits in the system except one are in the state $|0\rangle$.

On the other hand, it has been conjectured by several authors that entanglement can play

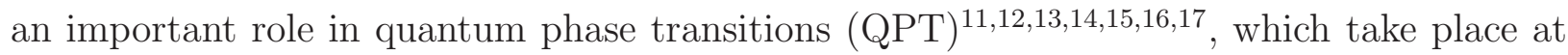
absolute zero temperature $\frac{18}{}$. For example, Osborne et al. $\stackrel{17}{\underline{7}}$ have stated that in QPT, longrange correlations take place due to entanglement. In addition, Osterloh et al $\stackrel{11}{\frac{11}{n}}$ analyzed the behavior of entanglement near the critical point of the spin 1/2 model XY in a transverse magnetic field and found that in the region close to a quantum phase transition the derivatives of entanglement of formation (concurrence) obey a scaling law. Thus, there exists an intimate connection between entanglement, scaling and universality. Further, quantum entanglement has been used not only near quantum phase transitions to characterize them, 
but also to obtain a better description of experimental measurements of specific heat and magnetic susceptibility of dilute solution of Ising dipoles $\frac{19}{}$. Additionally, we have recently shown that, in the case of rings with off-diagonal disorder, an increase in disorder strength results in enhancement of the nearest-neighbor (NN) concurrence with respect to the perfectly ordered ring 20 . This enhancement of concurrence hints that quantum entanglement might be a good indicator of anomalies in the wave functions and density of states in systems where no quantum phase transitions take place.

In the present paper, we report maximization of the concurrence using a computational optimization method. The calculations were done on the basis of an electronic model Hamiltonian which contains parameters depending on the lattice structure. Such parameters were optimized in order to maximize the concurrence. This means that, in general, the systems considered will not be translationally invariant after optimization, since the structure can be dramatically changed by the genetic algorithm. We present, specifically, calculations on systems described by a tight-binding model in one and two dimensional lattices. Our results can serve as a basis for the design of structures which maximize nearest-neighbor entanglement.

This paper is organized in the following manner: in section II the Hamiltonian is presented, as well as the most important formulas used to quantify concurrence and the key steps in the optimization procedure. Results for one and two dimensional bipartite lattices, as well as non-bipartite two dimensional lattices will be presented in section III. Finally, a summary of our results can be found in section IV.

\section{THEORY}

We consider electronic systems described by a tight-binding Hamiltonian of the form

$$
\hat{H}=\sum_{\langle i j\rangle} t_{i j} \hat{c}_{i}^{+} \hat{c}_{j}
$$

For simplicity, we consider spinless electrons. In Eq. (11) $\hat{c}_{i}^{+}\left(\hat{c}_{i}\right)$ is the usual creation (annihilation) operator of a spinless electron at site $i$ and $t_{i j}$ is the hopping integral between NN sites $i$ and $j$.

It should be noted that the magnitude of the hopping elements $t_{i j}$ can be easily related to the lattice structure. A large $t_{i j}$ indicates a small interatomic distance, which causes a 
large overlap between the wave functions localized on the sites $i$ and $j$. Analogously, a small $t_{i j}$ represents a large interatomic distance. $t_{i j}=0$ means that sites $i$ and $j$ are not nearest neighbors.

To calculate the entanglement of formation between pairs of sites we employed the concept of concurrence $\underline{\underline{10}}$ and proceeded in a similar way as Zanardi and coworkers did for the case of translationally invariant chains 21 .

For a system described by the Hamiltonian of Eq. (1), the concurrence between sites $i$ and $j$ has the form $\underline{5}$

$$
\mathrm{C}_{i j}=2 \max \{0,|z|-\sqrt{v y}\}
$$

where

$$
\begin{aligned}
& v=1-\left\langle\hat{n}_{i}\right\rangle-\left\langle\hat{n}_{j}\right\rangle+\left\langle\hat{n}_{i} \hat{n}_{j}\right\rangle \\
& y=\left\langle\hat{n}_{i} \hat{n}_{j}\right\rangle \\
& z=\left\langle\hat{c}_{j}^{+} \hat{c}_{i}\right\rangle .
\end{aligned}
$$

Note that $i$ and $j$ do not need to be nearest neighbors.

To maximize NN concurrence, we use genetic algorithms (GAs), a technique developed by J. H. Holland and his students at the University of Michigan in the 1960s and 1970s. Holland's goal was not to design algorithms to solve specific problems, but rather to formally study the phenomenon of adaptation as it occurs in nature and to develop ways in which the mechanisms of natural adaptation can be used for numerical optimization.

In this optimization technique, the characters or numbers representing the solution to a problem are stored in a string called "chromosome". Nature-inspired operators of crossover and mutation are applied to a population of chromosomes and those individuals that represent the best solution to the problem are given more probability of being chosen for the next generation. By iterating this process, it is possible to obtain a very good solution without having to explore the entire solution space.

For the particular problem treated in this paper, we propose first families of hopping integrals $\left\{t_{i j}\right\}$, which are then fine-tuned by means of the genetic algorithm. Our goal is to obtain the hopping elements from the Hamiltonian such that its ground state function represents the maximum average NN concurrence. 
We have used genetic algorithms in a previous paper ${ }^{22}$, where we have optimized the total concurrence $\left(\mathrm{C}_{\text {total }}\right)$ of one dimensional chains and two dimensional lattices described by a tight-binding Hamiltonian with open and periodic boundary conditions. $\mathrm{C}_{\text {total }}$ was taken as an average over all concurrences between every site $i$ with the $N-1$ remaining sites.

In this paper, however, we focus only on optimization of first-neighbor concurrence, which can be calculated by means of the following expression:

$$
\mathrm{C}_{\mathrm{NN}}=\frac{1}{z N} \sum_{i=1}^{N} \sum_{j=1}^{z} \mathrm{C}_{i j} .
$$

where $z$ is the number of nearest neighbors. $\mathrm{C}_{\mathrm{NN}}$ is our fitness function for the application of the genetic algorithm.

It is important to point out that a system with translational invariance is described by all the $t_{i j}$ elements from Eq. 1 having the same value, for example, $t_{i j}=-1$. This configuration leads to concurrence values that are the same for each pair of nearest neighbors, a case which has been previously studied by several authors $5,20,21$.

The genetic pseudo-algorithm employed in this work has been given in detail elsewhere ${ }^{22}$. Briefly, it consists in the following steps:

1. We consider each $t_{i j}$ of the Hamiltonian matrix as a gene and the array of these genes as a chromosome.

2. Allocate two arrays, "generation0" and "generation1" composed of chromosomes.

3. Allocate a chromosome "best" with fitness 0.0 .

4. For a given band filling, we repeat the following steps:

- Initialize "generation0" with random values in the range $(-5,0)$.

- For a given number of generations repeat:

- Decode each chromosome in "generation0" into a Hamiltonian matrix, diagonalize it and calculate the average concurrence between only nearest neighbors of the system using Eq. 6. In other words, we calculate the fitness $\mathrm{C}_{\mathrm{NN}}$ for each individual in "generation0".

- Compare "best" with the fittest individual in "generation0" and substitute "best" if the latter has larger fitness. 
- Choose chromosomes with a probability proportional to its fitness (selection operator) and copy it to "generation1".

- Use crossover and mutation operators on chromosomes in "generation1" to create new chromosomes.

- Make "generation0" equal to "generation1".

- Print "best" in an output file.

\section{RESULTS AND DISCUSSION}

In this section, we present our results of NN concurrence optimization using GAs. The effect of bipartite and non-bipartite systems with periodic boundary conditions is addressed. We recall that a lattice is considered bipartite if it can be separated in two sublattices $A$ and $B$ such that all first neighbors of the sites of sublattice $A$ are sites from sublattice $B$ and vice versa. Biparticity or non-biparticity has important consequences in the physical properties of a lattice. For example, the concurrence of a bipartite lattice is symmetric around half band filling while non-biparticity is responsible for magnetic frustration in spin systems, that is, the impossibility of minimizing energy for each pair of spins in the lattice.

\section{A. Bipartite Systems}

First, we focus on finite one dimensional chains of up to $N=50$ sites with periodic boundary conditions. Results are shown in Fig. 1. We considered 500 chromosomes (i.e. a collection of hopping integrals from the hamiltonian matrix) in each population, with starting values randomly chosen in the range $(-5,0)$. The algorithm was left to evolve 4000 generations in order to obtain the individuals with the best fitness value. This procedure was undertaken for each band filling $(x=n / N)$. From the figure it can be seen that there exists symmetry around half band filling, indicating that it is possible to focus only on the $0 \leq x \leq 0.5$ region when analyzing concurrence in bipartite lattices.

Optimized concurrence shows two distinctive features: (a) the growth of concurrence as function of band filling is quasi-linear for $x \leq 0.25$. This result is due to the fact that in lower band fillings, the probability of electronic collision is low, and the system is able to optimize

concurrence without considering Pauli repulsion. (b) For $x>0.25$ the growth of $\mathrm{C}_{\mathrm{NN}}$ as 
function of $x$ is quasi-parabolic, and electronic exchange due to the Pauli principle comes into play. Comparison between results obtained using GAs and the ordered case -with all $t_{i j}$ taken equal- shows that for low band filling $(x \leq 0.1)$, both concurrence curves display a similar behavior and the impact of optimization starts to be noticeable for $x>0.1$, with a maximum increase in concurrence with respect to the ordered case of $47 \%$ (0.5 vs 0.3392 ) at half band filling.

In order to understand the behavior of optimized concurrence, we have analyzed those chromosomes with best fitness values for each $x$. Results show that, as band filling $x$ is increased, the ring starts to break into smaller chains (i.e., some hopping elements become $t_{i j} \simeq 0$ ). For example, while for $n=1$ all $t_{i j}$ are similar and close to -4 , in the next filling, $n=2$, two noncontiguous elements $t_{i j}$ differ from the rest and are now close to 0 , suggesting a separation into two subchains. This division increases with $x$ until half band filling $(x=0.5)$, a point at which the system alternates a short bond $\left(t_{i j} \simeq-5\right)$ with a long one $\left(t_{i j} \simeq 0\right)$ as can be seen depicted in Fig. 2. In other words, to increase the average concurrence, the system begins to dimerize, i.e, the chain undergoes a structural phase transition due to a Peierls instability $23,24,25$. This is a metal-insulator transition occurring in one-dimensional metals, where the doubling of the unit cell leads to a decrease in the kinetic energy of the system. Note that dimerization of the chain was obtained in a natural manner, since all initial matrix elements were initialized by random values. In fact, the maximum concurrence at half band filling corresponds to a system which has completely dimerized. This shows a possible connection between the increase in concurrence and the Peierls instability. In order to study this connection, we analyzed the concurrence and their derivatives for a dimerized chain. In this case, we consider that the nearest-neighbor hopping integrals $t_{i j}$ of Eq. 1 take the values $t_{2 n, 2 n+1}=1+\alpha$ and $t_{2 n-1,2 n}=1-\alpha$, where $\alpha \in[0,1]$ is the dimerization parameter. This one-particle dimerized Hamiltonian can be diagonalized by using the transformations proposed by Su, Schrieffer and Heeger 24 . The goal of analyzing the concurrence and its derivative is based on the fact that a discontinuity (singularity) in the (derivative) groundstate concurrence has been associated to a first (second) order QPT $\underline{11}, \underline{12}, \underline{13}, \underline{14}, \underline{15}$. However, it has been shown that the relation between QPT and non-analyticity in the concurrence is not one-to-one $\frac{13}{}$. A one-to-one connection can be assumed though, when we consider QPTs characterized by nonanalytic behavior in the derivatives of the ground-sate energy and we exclude artificial and accidental occurrences of non-analyticities in the ground-state 
concurrence and its derivative $\stackrel{14}{\underline{4}}$. In Fig. 3, we present the ground-state concurrence of a dimerized chain, $\mathrm{C}_{2 n, 2 n+1}$ and $\mathrm{C}_{2 n-1,2 n}$, and its derivative, $\mathrm{C}_{2 n, 2 n+1}^{\prime}$ and $\mathrm{C}_{2 n-1,2 n}^{\prime}$, where $\mathrm{C}^{\prime}$ means $d \mathrm{C} / d \alpha$, as a function of $\alpha$. Note that $\mathrm{C}_{2 n, 2 n+1}=2.0 * \max \left\{0, \gamma_{2 n, 2 n+1}+\gamma_{2 n, 2 n+1}^{2}-0.25\right\}$ and $\mathrm{C}_{2 n-1,2 n}=2.0 * \max \left\{0, \gamma_{2 n-1,2 n}+\gamma_{2 n-1,2 n}^{2}-0.25\right\}^{20}$, where $\gamma_{2 n, 2 n+1}=\left\langle c_{2 n}^{\dagger} c_{2 n+1}\right\rangle$ and $\gamma_{2 n-1,2 n}=\left\langle c_{2 n-1}^{\dagger} c_{2 n}\right\rangle$ are the one-particle density-matrix elements or bond orders between NN and can be calculated analytically 24 . These bond orders are continuous functions of $\alpha$, the first one ranging from $\gamma_{2 n, 2 n+1}=0.318310(\alpha=0)$ to $0.5(\alpha=1.0)$ and the second one from $\gamma_{2 n-1,2 n}=0.318310(\alpha=0)$ to $0.0 . \quad(\alpha=1.0)$. Therefore, the discontinuity obtained for $\mathrm{C}_{2 n-1,2 n}$ at $\alpha \approx 0.138$ is not related to a critical point. Clearly, this discontinuity is artificial and comes from the particular definition of the concurrence in Eq. (2) $)^{13,14}$.

In Fig. 3 one observes that $\mathrm{C}_{2 n, 2 n+1}^{\prime}$ and $\mathrm{C}_{2 n-1,2 n}^{\prime}$ present singularities at the limit $\alpha \rightarrow$ $\alpha_{c}=0$, where the Peierls instability occurs, which should be related with a second order $Q P T \stackrel{14}{ }$. In order to investigate if the singularity is related with a second order QPT, we write the ground state energy (and their derivatives) as a function of the NN densitymatrix elements (and their derivatives). The ground-state energy per site is given by $E_{\mathrm{gs}}=$ $-(1+\alpha) \gamma_{2 n, 2 n+1}-(1-\alpha) \gamma_{2 n-1,2 n}$, its first derivative by $d E_{\mathrm{gs}} / d \alpha=-(1+\alpha) d \gamma_{2 n, 2 n+1} / d \alpha-$ $(1-\alpha) d \gamma_{2 n-1,2 n} / d \alpha-\gamma_{2 n, 2 n+1}+\gamma_{2 n-1,2 n}$ and its second derivative by $d^{2} E_{\mathrm{gs}} / d^{2} \alpha=-(1+$ $\alpha) d^{2} \gamma_{2 n, 2 n+1} / d^{2} \alpha-(1-\alpha) d^{2} \gamma_{2 n-1,2 n} / d^{2} \alpha-2 d \gamma_{2 n, 2 n+1} / d \alpha+2 d \gamma_{2 n-1,2 n} / d \alpha$. These second derivatives presents a singularity at $\alpha_{c}$, which is a manifestation of a second order QPT.

On the other hand, the maximal value of the $\mathrm{C}_{\mathrm{NN}}$ is obtained when the chain is completely dimerized $(\alpha=1)$, i.e. the chain has been transformed into $N / 2$ singlet Bell states. This complete dimerization can be related to the ideas of monogamy between three systems ${ }^{9}$ : the optimized system prefers to have maximum concurrence $(\mathrm{C}=1)$ between subsystems $A$ and $B$ while lowering that between $B$ and $C(\mathrm{C}=0)$.

The effect of dimensionality over $\mathrm{C}_{\mathrm{NN}}$ is shown in Fig. 4, where calculations for a square lattice $6 \times 6$ using a population of 700 individuals were performed. We considered 10,000 generations for each band filling. From the figure, it is possible to observe again two clearly remarkable traits for two ranges of $x$, as in the one dimensional case: a low band filling range $(x \leq 0.25)$ where the increase of $\mathrm{C}_{\mathrm{NN}}$ as function of $x$ is quasi-linear due to low collision probability between particles, and a second range $x>0.25$ where the Pauli exclusion principle starts to play an important role. $\mathrm{C}_{\mathrm{NN}}$ as function of $x$ has a quasi-parabolic behavior in the latter range, decreasing first in the range $0.25<x \leq 0.375$ and then increasing 
for $0.375<x \leq 0.5$. It is also noteworthy that both regions have the same maxima of $\mathrm{C}_{\mathrm{NN}} \approx 0.25$, one at $x=0.25$ and the other at $x=0.5$. Comparison of $\mathrm{C}_{\mathrm{NN}}$ between the optimized case and an ordered, large lattice shows that the greatest difference occurs at half band filling. Moreover, we observe that maxima $\mathrm{C}_{\mathrm{NN}}$ obtained for the square lattice is smaller than the maximum $\mathrm{C}_{\mathrm{NN}}$ for the $1 \mathrm{D}$ ring.

A study of the fittest chromosomes for those band fillings where $\mathrm{C}_{\mathrm{NN}}$ is a maximum shows the following behavior: for $x=0.25$, the short bonds $\left(t_{i j} \simeq-5\right)$ form nine squares which are separated by long bonds $\left(t_{i j} \simeq 0\right)$ (Fig. $5(\mathrm{a})$ ). In each square, a spinless electron can be found. This result is related to the definition of generalized $\left|W_{N}\right\rangle$ states, $\left|W_{N}\right\rangle=(1 / \sqrt{N})|N-1,1\rangle$ where the state $|N-1,1\rangle$ denote the totally symmetric state with $N-1$ zeros and one 1 . It has been shown that these $\left|W_{N}\right\rangle$ states are very robust against particle losses and that the concurrence of two qubits can be determined to be $\mathrm{C}_{i j}=2 / N^{\underline{7}}$. In the case of $N=4$, we have that $\left|W_{4}\right\rangle=(1 / \sqrt{4})[|0001\rangle+|0010\rangle+|0100\rangle+|1000\rangle]$ and $\mathrm{C}_{i j}=0.5$ for two NN sites of a square. Using this $\mathrm{C}_{i j}$ value for two $\mathrm{NN}$ sites of a square, it is easy to show that $\mathrm{C}_{\mathrm{NN}}=0.25$ (see Eq. 6). On the other hand, for $x=0.5$ the system tries once again to form dimerized states, separated with long bonds (Fig. 5(b) . The decrease of $\mathrm{C}_{\mathrm{NN}}$ with respect to the one dimensional ring is related to the difference in the number of neighbors between both systems. In the one dimensional systems, $\mathrm{C}_{\mathrm{NN}}$ is obtained by averaging between two nearest-neighbors whereas in the square lattice this average requires four nearest-neighbors.

\section{B. Non Bipartite Systems}

In order to study the effect of non-biparticity on the concurrence, we have considered three non-bipartite lattices: the Kagomé lattice, the maple leaf or Betts lattice and the triangular lattice. Note that the triangular lattice is less bipartite than the two other ones due to its larger number of triangular bonds. This has important consequences in antiferromagnetic systems $\underline{26}$.

The basic common feature of the results presented so far is that successful individuals in the linear chain and square lattices form open and tightly closed binary subsystems. Thus, we exploited this fact in order to speed up the procedure and increase the accuracy of the GA calculations. We initialized a hundred individuals of the population to 0.0 and a hundred to -5.0. The motivation for performing this step is that this short and long bonds will spread 
to other individuals through the crossover operator, and that this kind of bonds will help increase its concurrence.

We now apply our calculations to a periodic Kagomé lattice of 48 sites with periodic boundary conditions. Results are shown in Fig. 6. We considered 1200 individuals in the population, which was left to evolve over 3000 generations. Quasi-linear behavior of $\mathrm{C}_{\mathrm{NN}}$ with $x$ can be seen in regions with low electronic or hole density, namely $x \leq 0.2$ and $x \geq 0.8$. This behavior corresponds to low probability of collision, as has already been discussed, and is independent from the kind of lattice or whether it presents triangular bonds or not. The first effect of non-biparticity is reflected in the lack of symmetry of $\mathrm{C}_{\mathrm{NN}}$ around half band filling. Note that Fig. 6 presents only one distinguishable maximum located -contrary to the case bipartite lattices-, out of half band filling, at $x \simeq 1 / 3$. Comparison with respect to the ordered case shows the relevance of the optimization technique, particularly for $0.6 \leq x \leq 0.8$, where the initially ordered structure displays a very low concurrence. Moreover, there is a coincidence in the localization of the maximum for both the optimized and ordered lattices. The best individual corresponding to the maximum of the numerical optimization approach can be seen in Fig. 7(a), where one can observe that a geometrical configuration favoring triangular loops has been formed. It is clear again that this trend to form triangular loops is related to $W$ states, $|W\rangle=(1 / \sqrt{3})[|100\rangle+|010\rangle+|001\rangle]$, where $\mathrm{C}_{i j}=2 / 3$ for two NN sites forming the triangle. To further extend the optimized results, we now introduce a design as seen in Fig. 7(b). A new concurrence curve for the Kagomé lattice is presented in Fig. 6. In this case, besides the hundred individuals initialized to 0.0 and -5.0 each, all other individuals were initialized with the proposed design of Fig. 7(b), As can be seen, the concurrence is not only increased at the maximum, but the proposal improved the overall behavior of the curve as well.

Considering the hint given by the analysis of the Kagomé lattice, we ran the optimization algorithm for the maple-leaf or Betts lattice in order to find an optimum structure design. Fig. 8 shows the best individual of an initial run of the algorithm corresponding to the maximum at $x=0.33$ [Fig. 8(a)], as well as a proposal based on this individual [Fig. 8(b)]. Fig. 9 shows the results for the ordered case, as well as both runs of the algorithm. As expected, the ordered case performed worse than any of the optimized cases. Once again, the run based on proposal shown in Fig. 8(b)] performed better than the random-based case.

Finally, in the case of the triangular lattice, the typical behavior found in the other non- 
bipartite lattices can be readily observed: a single maximum located near $x=1 / 3$ and a much better performance with respect with the ordered case (see Fig. 101). As in the other cases, we then proceeded to develop new proposed configurations by looking at successful individuals and being inspired by $W$ states. Two new designs were chosen, seen in Figures $11(\mathrm{a})$ and $11(\mathrm{~b})$.

The best curve for $\mathrm{C}_{\mathrm{NN}}$ can be seen in Fig. 10, and is basically a combination of the different curves obtained when initializing with each proposal. Although there is a fair increase in the overall maximum $\left(\mathrm{C}_{\mathrm{NN}}=0.2222\right)$, it is still lower than that of the Kagomé lattice $\left(\mathrm{C}_{\mathrm{NN}}=0.3333\right)$ and maple leaf lattice $\left(\mathrm{C}_{\mathrm{NN}}=0.2666\right)$. This is because in the triangular lattice there are more bonds, and consequently each site must share its entanglement with more neighbors.

\section{SUMMARY AND CONCLUSIONS}

In this work, we have used GAs to maximize the nearest-neighbor average concurrence of systems by tuning the nearest-neighbor hopping integrals of a tight binding Hamiltonian. The optimization of entanglement has been performed for one- and two dimensional bipartite systems as well as for two dimensional non bipartite systems. The results show that the concurrence of the optimized systems is very large in comparison with the ordered structures. This increase in the concurrence is understood and interpreted by analyzing the optimized nearest-neighbor hopping integrals. In general, we found certain tendencies of periodical systems to break into smaller subsystems. This is achieved in a natural manner by the system by making the hopping integrals evolve in such a way that the absolute value of some integrals is high in some cases $\left(\left|t_{i j}\right| \simeq 5\right)$ and very small in others $\left(t_{i j} \simeq 0\right)$.

This results are related to the fact that quantum entanglement, in contrast with classical correlations, cannot be freely shared between many objects. This quantum correlations property - monogamy - , is clearly noticeable in the case of the periodic ring at $x=0.5$, where the $n$-th site is completely entangled with the site $n+1\left(\mathrm{C}_{\mathrm{n}, \mathrm{n}+1} \simeq 1\right)$ while on the

other hand it is completely unentangled with the site $n-1\left(\mathrm{C}_{\mathrm{n}, \mathrm{n}-1} \simeq 0\right)$. Moreover, results at $x=0.5$ shows that, in order to maximize concurrence, the system undergoes a structural transition, the Peierls distortion.

Finally, it is worth mentioning that the relationship between the 1D tight binding Hamil- 
tonian [Eq. (1)] and the $X X$ chain of $1 / 2$ spin given by the following expression

$$
\hat{H}_{X X}=\sum_{j}^{N_{a}} \frac{J_{j}}{2}\left(S_{j+1}^{+} S_{j}^{-}+S_{j}^{-} S_{j+1}^{+}\right),
$$

where $J_{j}$ is the coupling constant. This relationship can be shown by using the JordanWigner transformation $\frac{18}{}$ and therefore, it can be inferred that our results for the 1D tight binding systems can be used to describe the maximization of the $\mathrm{C}_{\mathrm{NN}}$ concurrence for systems modeled by the $X X$ chain of $1 / 2$ spin.

\section{Acknowledgments}

We thank CONACYT for providing support for one of the authors (J.C.N.M). The computational resources that we used for this work were provided by the National Center of Supercomputing CNS-IPICyT, Mexico.

1 C. H. Bennett, G. Brassard, C. Crepeau, R. Jozsa, A. Peres and W. K. Wootters, Phys. Rev. Lett. 70, 1895 (1993).

2 C. H. Bennett, S. J. Wiesner, Phys. Rev. Lett. 69, 2881 (1992).

3 C. H. Bennett and G. Brassard, Proceedings of IEEE International Conference on Computers,

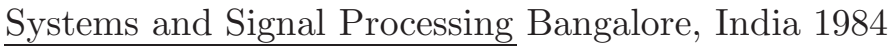

4 M. A. Nielsen and I. L. Chuang, Quantum Computation and Quantum Information (Cambridge University Press, Cambridge, United Kingdom,2000).

5 K. M. O'Connor and W. K. Wootters, Phys. Rev. A 63, 052302 (2001).

6 W. K. Wootters, e-print arXiv quant-ph/0001114.

7 W. Dür, G. Vidal, and J. I Cirac, Phys. Rev. A 62, 062314 (2000).

8 M. Koashi, V. Buzek and N. Imoto, Phys. Rev. A 62, 050302(R) (2000).

9 V. Coffman, J. Kundu and W. K. Wootters, Phys. Rev. A 61, 052306 (2000).

10 W. K. Wootters, Phys. Rev. Lett. 80, 2245 (1998); S. Hill and W. K. Wootters, Phys. Rev. Lett. 785022 (1997).

11 A. Osterloh, L. Amico, G. Falci and R. Fazio, Nature 416, 608 (2002).

12 S.J. Gu, H. Q. Lin, and Y. Q. Li, Phys. Rev. A 68, 042330 (2003). 
13 M.-F. Yang Phys. Rev. A 71, 030302(R) (2005).

14 L.-A. Wu, M. S. Sarandy and D. A. Lidar, Phys. Rev. Lett. 93, 250404 (2004).

15 D. Larsson and H. Johannesson, Phys. Rev. Lett. 95, 196406 (2005).

16 G. Vidal, J. I. Latorre, E. Rico and A. Kitaev, Phys. Rev. Lett. 90, 227902 (2003).

17 T. J. Osborne and M. A. Nielsen, e-print arXiv quant-ph/0109024

18 S. Sachdev, Quantum Phase Transitions, (Cambridge University Press, Cambridge, United Kingdom,2001)

19 S. Ghosh, T. F. Rosenbaum , G. Aeppli and S. N. Coppersmith, Nature 425, 48 (2003).

20 R. Lopez-Sandoval and M. E. García, Phys. Rev. B 74, 174204 (2006).

21 P. Zanardi and X. Wang J. of Phys. A 35, 7947 (2002).

22 J. C. Navarro-Muñoz, H. C. Rosu, R. López-Sandoval Phys. Rev. A 74, 052308 (2006).

23 R. E. Peierls, Quantum Theory of Solids (Clarendon, Oxford, United Kingdom, 1955).

24 W. P. Su, J. R. Schrieffer, and A. J. Heeger, Phys. Rev. B 22, 2099 (1980).

25 Peter Fulde, Electron Correlations in Molecules and Solids (Springer-Verlag, Berlin, Germany, 1993).

26 G. Aeppli and P. Chandra, Science 275, 177 (1997).

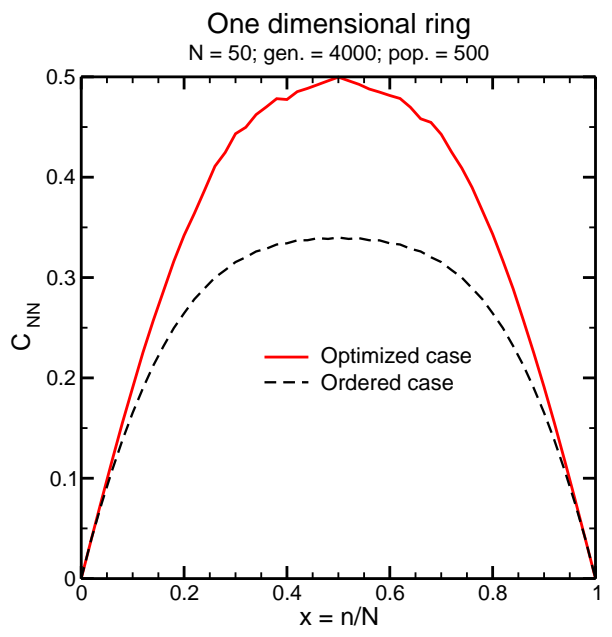

FIG. 1: (Color online) Nearest-neighbor concurrence $\mathrm{C}_{\mathrm{NN}}$ of chains with periodic boundary conditions as a function of band filling $x=n / N$. 


\section{0}

FIG. 2: (Color online) Graphical representation of a one-dimensional ring with 32 sites in the lattice structure yielding the maximal nearest-neighbor concurrence (see text). Parameters: 5000 generations; population $=600$. Half band filling. Three cases are distinguished by color: light grey $\left(\left|t_{i j}\right|<1\right)$, grey $\left(1 \leq\left|t_{i j}\right|<3\right)$ and black $\left(3 \leq\left|t_{i j}\right|<5\right)$.
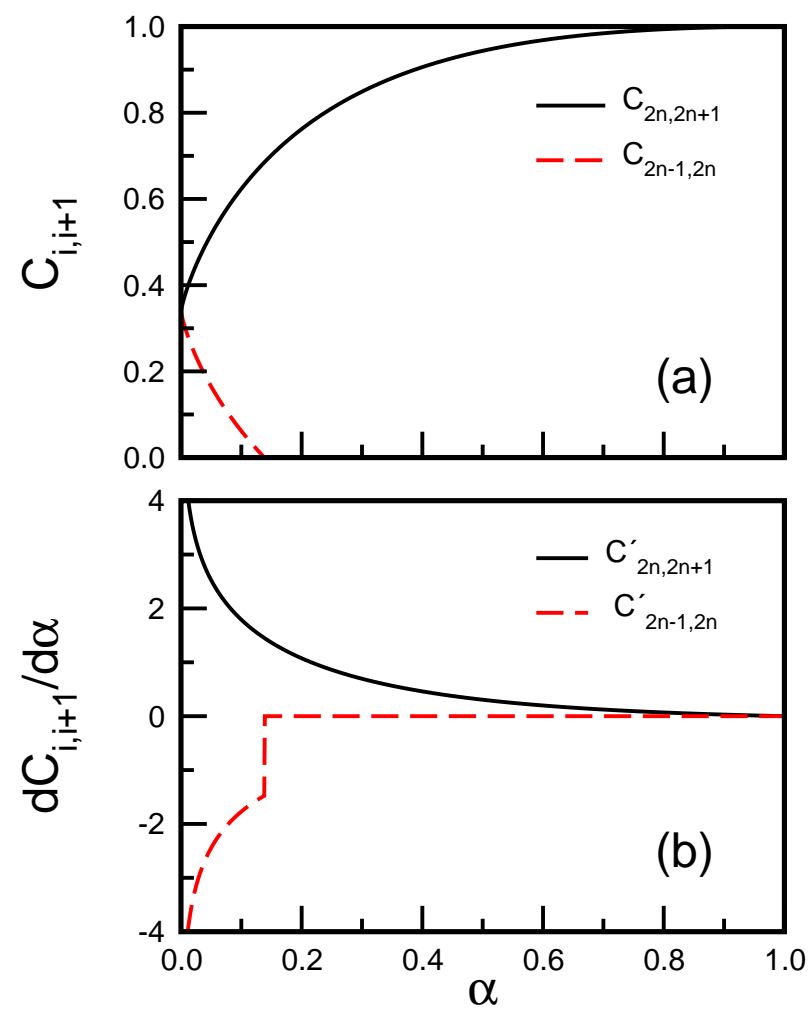

FIG. 3: (Color online) (a) Nearest-neighbor ground-state concurrences $\mathrm{C}_{i, i+1}$ of the dimerized chain (b) and their derivatives as a function of the dimerization parameter $\alpha$. 


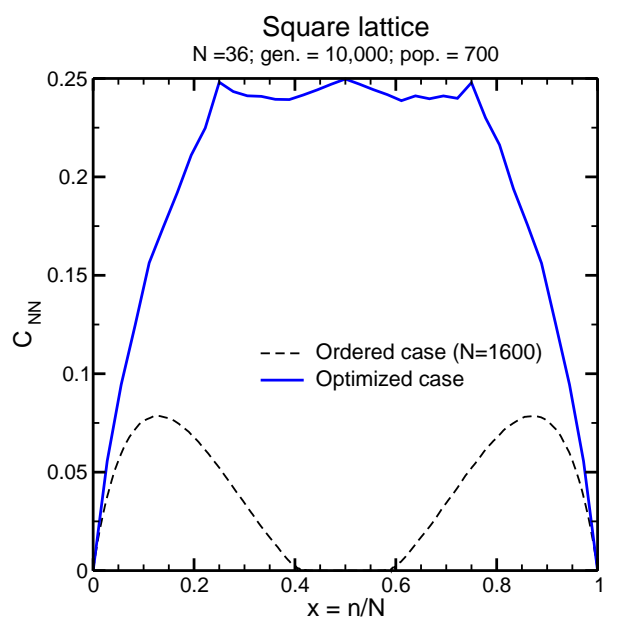

FIG. 4: (Color online) Nearest-neighbor concurrence $\mathrm{C}_{\mathrm{NN}}$ of a $6 \times 6$ square lattice as a function of band filling $x$. The solid line refers to the optimized system. The dashed line shows the concurrence of a large perfectly periodic square lattice $(N=1600)$.

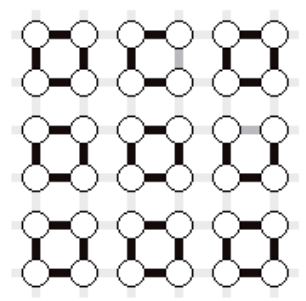

(a)Band filling $=$

$25 \%$

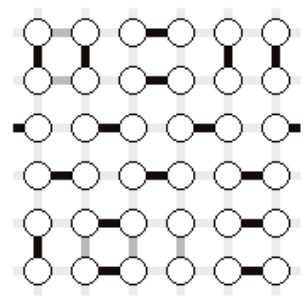

(b)Band filling $=$

$50 \%$

FIG. 5: Graphical representation of the optimized structures obtained starting from a square lattice of $36(6 \times 6)$ sites at (a) a quarter band filling and (b) half band filling. Parameters: 10000 generations; population $=700$. Three cases are distinguished by color: light grey $\left(\left|t_{i j}\right|<1\right)$, grey $\left(1 \leq\left|t_{i j}\right|<3\right)$ and black $\left(3 \leq\left|t_{i j}\right|<5\right)$. 


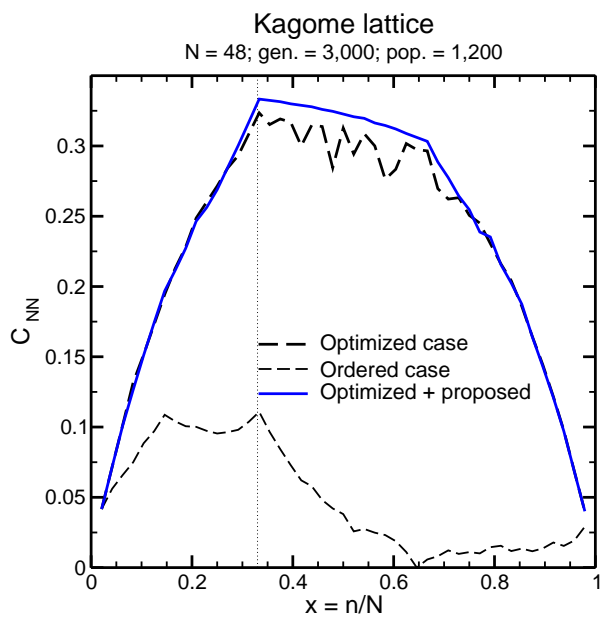

FIG. 6: (Color online) Nearest-neighbor concurrence $\mathrm{C}_{\mathrm{NN}}$ of small periodic Kagomé lattice as a function of band filling $x$. In addition, nearest neighbor concurrence $\mathrm{C}_{\mathrm{NN}}$ for the Kagomé lattice were calculated using the proposed optimal structure of Fig. 7(b)

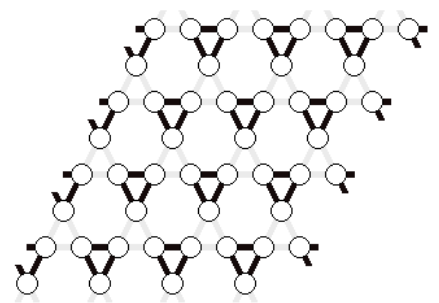

(a)Best individual for

Kagomé lattice

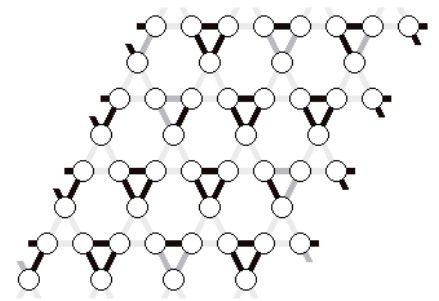

(b)Proposed optimal

structure

FIG. 7: Graphical representation of a Kagomé lattice of 48 sites. (a) represents the best individual using genetic algorithms at $x=33 \%$. (b) depicts a proposed optimal structure based on (a).

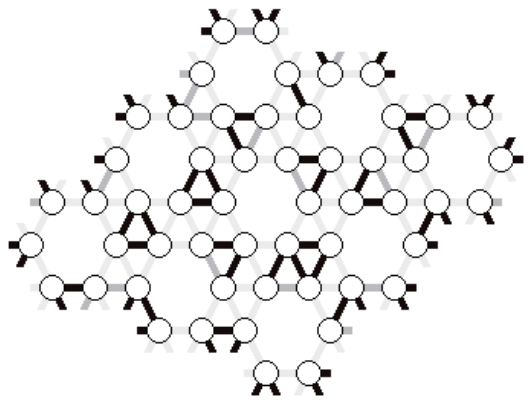

(a)Best individual for Betts lattice

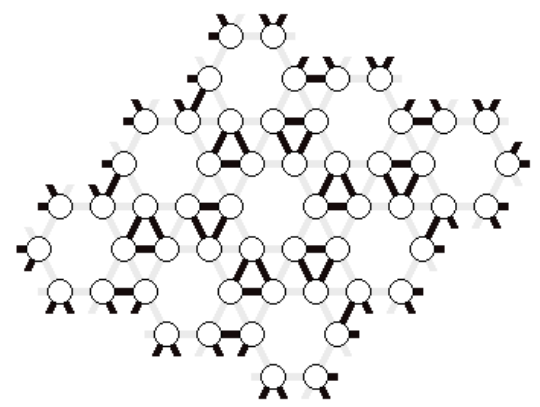

(b)Proposed optimal structure

FIG. 8: Graphical representation of a Betts lattice of 48 sites. (a) represents the best individual using genetic algorithms at $x=33 \%$. (b) depicts a proposed optimal structure based on (a). 


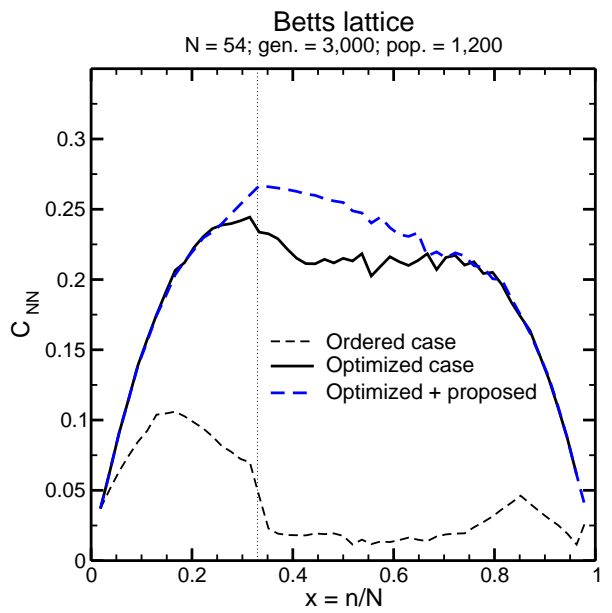

FIG. 9: (Color online) Nearest-neighbor concurrence $\mathrm{C}_{\mathrm{NN}}$ for the Betts lattice as a function of $x$. The figure shows $\mathrm{C}_{\mathrm{NN}}$ for the ordered case, the optimized case using genetic algorithms and, finally, the case where the generations were initialized at each band filling using the proposed optimal structure of Fig. 8(b).

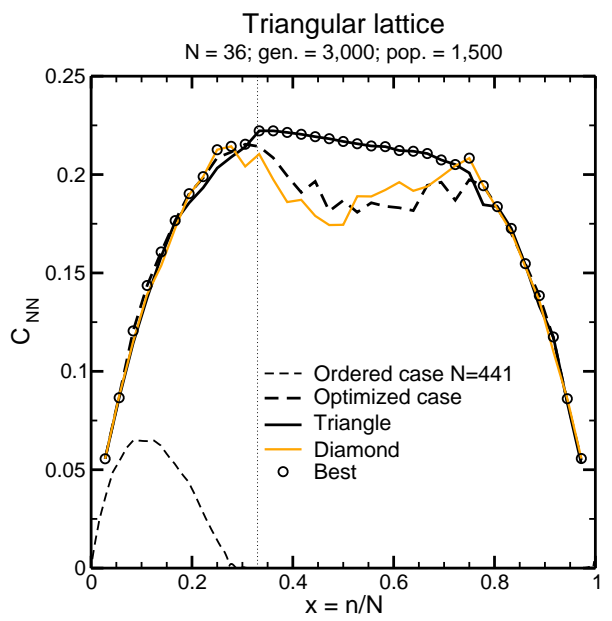

FIG. 10: (Color online) Nearest-neighbor concurrence $\mathrm{C}_{\mathrm{NN}}$ for the triangular lattice as a function of $x$. The nearest-neighbor concurrence $\mathrm{C}_{\mathrm{NN}}$ was calculated for the ordered case, the optimized case using genetic algorithms, the proposed optimal structures and the case combining results from proposals. 


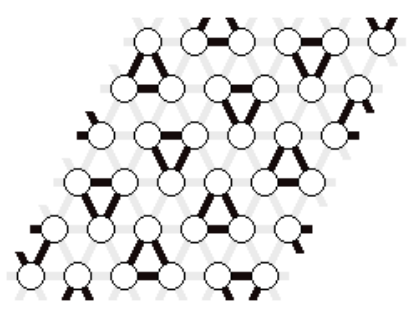

(a)Proposed optimal structure "Triangle"

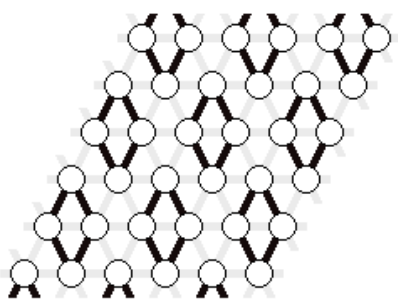

(b)Proposed optimal

structure "Diamond"

FIG. 11: Proposed optimal structures based on the best individual obtained using genetic algorithms. The "triangle" structure is based on the best individual at $x=33 \%$ whereas the diamond is inpired on the best individual at $x=0.69$. 